\title{
Towards Sensor Failure Detection in Ambient Assisted Living: Sensors Correlations
}

\author{
Nancy E. ElHady and Julien Provost
}

\begin{abstract}
Ambient Assisted Living promotes healthy independent ageing of the elderly at their homes by monitoring their behaviour, and support medical assistance whenever needed. For privacy and acceptance issues, non-intrusive sensors are preferably used. However, such sensors are more prone to produce false positive or negative data. Faulty sensor data could be automatically detected if correlations between sensors can be identified. This paper aims to propose the use of association rule mining to find correlations between binary event-driven sensors installed for monitoring purposes in an apartment. A case study was carried out to validate the approach and investigate the effect of different data mining parameters on the quality of obtained association rules. The results show that correlations could be successfully deduced from unlabelled datasets with no prior expert knowledge on the sensors topology.
\end{abstract}

\section{INTRODUCTION}

The Ageing population phenomenon is affecting countries all over the world, with an expectancy of multiplying by two the number of people aged over 60 years by 2050 . Several countries will have more than $30 \%$ of its population over 60 years old, like Germany, France, China, Canada and others [1]. To be able to face this demographic shift, an increasing numbers of research works are investigating the development of approaches and tools for Ambient Assisted Living (AAL). Ambient Assisted Living promotes healthy ageing in the elderly's place of residence by using information and communication technologies to monitor their Activities of Daily living (ADL), detect deviation of their behaviour, predict their future activities, and provide help whenever needed.

Sensors used to monitor the behaviour of elderly people at their homes are either intrusive sensors, e.g., cameras and microphones, or non-intrusive sensors, e.g., motion sensors and contact sensors. The systems equipped with intrusive sensors are not highly accepted by the population due to privacy and security concerns. Consequently, in the last decade, a stronger focus in research was directed towards the use of non-intrusive sensors in AAL. However, such sensors often suffer from false positive or negative triggers that can affect the performance of the system.

Two types of sensor failures could be encountered; failstop failures, where the sensors completely stop responding, and non-fail-stop failures, where the sensors are still working but give false information about their environment. The typical non-fail-stop sensors malfunctions that were reported by Flöck [2] during practical implementation of AAL include

Nancy E. ElHady and Julien Provost are with with Assistant Professorship of Safe Embedded Systems, Technical University of Munich, Garching, Germany nancy.elhady@tum. de; julien.provostetum.de spurious signals of motion sensors at night, faulty activation of motions sensors by sunlight, bouncing of door contact sensors for several minutes, and switch-off delays of motion sensors after the last observed activity. Other sources for non-stop-failures could be moved-location failure when the sensor gets remounted by the resident to another location, and obstructed-view failure where the sensor gets blocked by furniture [3].

Various fault detection techniques have been developed for wireless sensor networks that consist of homogeneous, timedriven and continuous-valued sensors, e.g., majority voting scheme and time-series analysis. However, the non-intrusive sensors used in AAL are heterogeneous, event-driven and binary sensors.

Sensor failure detection in Ambient Assisted Living equipped with non-intrusive ambient sensors has been approached previously in a few works.

SMART [3] used classification technique in which the classifier instances are trained with one sensor left out of the training dataset to replicate a sensor failure. This approach deals only with single sensor failure and lacks scalability due to the significantly increasing training effort required.

FailureSense [4] exploited the correlation between the turn on/off of electrical appliances and the sensors trigger events based on Gaussian mixture model. However, it assumes that the person has to be physically beside the electrical appliance to turn it on/off and the average failure detection latency is approximately 22 hours. A clustering based outlier detection was proposed in [5], that can only deal with false positives sensor triggers but not false negatives.

Amri et al. [6] used q-relaxed intersection technique to detect faulty sensors via comparing the estimated location of the resident from the activation of motion sensors with the location estimated from the random walk model. Nevertheless, the random walk model is not accurate enough to model the resident behaviour.

Idea's [7] approach to detect failures is based on the assumption that there are functional redundant sensors for ADL recognition. A sensor failure is flagged when the probability that a certain activity has been detected while the sensor was not triggered exceeds a certain threshold. This approach relies on accurate ADL recognition which can not be guaranteed in presence of sensor failures, in addition it needs labelled dataset for the training phase.

Ye et al. [8] have attempted to detect missing sensor data by finding correlations between sensors using mutual information technique along with predicting the trigger time using non-liner time series analysis techniques. However, the 
authors could not prove the effectiveness of their approach due to the limited duration of the testing dataset and the low number of used sensors beside its biased distribution across the flat under test.

As highlighted in the previous paragraph, sensor failures detection in AAL is still challenging, especially in the presence of the non-deterministic human behaviour that made model-based fault detection unable to guarantee good results. This paper aims to find correlations between non-intrusive binary sensors using a data-driven approach, specifically association rule mining. A refinement to the association rule mining method is proposed and a comparison of the results obtained for different parameters is done in order to evaluate the feasibility of extraction correlations. Those sensors correlations could be utilized in the future for the detection of fail-stop and non-fail-stop sensor failures in AAL.

\section{BACKGROUND}

In order to detect sensor failures in AAL, large sensors datasets have, first, to be thoroughly analysed to detect the fault-free sensors correlations during the nominal behavior of the resident. Association rule mining is a data mining technique that was introduced by Agrawal et al. [9] to find associations between items in large datasets. Association rule mining was successfully used in various fields, with its most common application is the market basket analysis [10].

The items in the datasets are the set of binary features denoted as $\mathrm{I}=\{\mathrm{I} 1, \mathrm{I} 2, . ., \mathrm{Im}\}$. The dataset consists of a number of transactions $\mathrm{T}=\{\mathrm{T} 1, \mathrm{~T} 2, . ., \mathrm{T} n\}$, where each transaction contains a subset of the items $\mathrm{I} ; \mathrm{T} \subseteq \mathrm{I}$. The association rule is in the form of $\mathrm{X} \rightarrow \mathrm{Y}$, where $\mathrm{X} \subset \mathrm{I}, \mathrm{Y} \subset \mathrm{I}$ and $\mathrm{X} \cap$ $\mathrm{Y}=\phi . \mathrm{X}$ and $\mathrm{Y}$ are the itemsets called the antecedent and consequent of a rule, respectively. An association rule $\mathrm{X} \rightarrow$ $\mathrm{Y}$ means that "IF the item(s) X occured THEN the item(s) Y occured as well". There are two important evaluation metrics for each rule, which are the support and confidence of this rule, defined as follows:

$$
\begin{aligned}
& \operatorname{Support}(X \rightarrow Y)=\frac{\mid \text { Transactions containing X\&Y } \mid}{\mid \text { Transactions } \mid} \\
& \text { Confidence }(X \rightarrow Y)=\frac{\mid \text { Transactions containing X\&Y } \mid}{\mid \text { Trasactions containing } \mathrm{X} \mid}
\end{aligned}
$$

To find the association rules of interest from a dataset, minimum support and confidence are predefined by the user for the association rule mining. The support reflects how likely it is to find the items of $\mathrm{X}$ and $\mathrm{Y}$ together in the transactions of a dataset, while the confidence reflects how frequent the items of $\mathrm{Y}$ in the transactions that contains items of $\mathrm{X}$.

One of the widely used algorithms for association rule mining is the Apriori algorithm, in which the dataset is first scanned to find 1-itemsets (itemsets of length 1) that satisfy the minimum support, then from those frequent 1-itemsets, 2itemsets will be generated and checked against the minimum support value, and so on [11]. Only the association rules

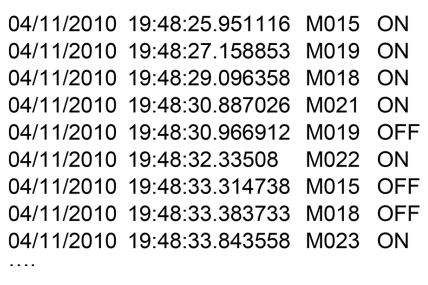

Fig. 1. Sample of the sensors dataset

that satisfy the minimum support and minimum confidence will be extracted. Another evaluation metric for association rules is the lift, which confirms the correlation between the antecedent and consequent items of rules if its value is greater than 1. The lift of an association rule is defined as follows:

$$
\begin{aligned}
& \operatorname{Lift}(X \rightarrow Y)= \\
& \frac{\mid \text { Transactions containing } \mathrm{X} \& \mathrm{Y} \mid}{\mid \text { Transactions containing } \mathrm{X}|*| \text { Transactions containing } \mathrm{Y} \mid}
\end{aligned}
$$

\section{APPROACH}

An essential step towards developing a sensor failure detection system is to find strong correlations between the employed sensors. In this paper, we investigate the use of association rule mining to find the highly correlated sensors from an unlabelled recorded dataset. The obtained rules could then be used for sensor failure detection in such a way that if the sensor(s) of the antecedent part of rule got triggered while the sensor(s) of the consequent part of rule did not within a specific time, then the sensor(s) can be suspected to be faulty. The higher the correlation, the higher the confidence in the sensor failure detection and the shorter the time to detection.

\section{A. Data Preprocessing}

As the association rule mining was primarily designed for transactional databases, some modifications had to be done so that this method would better suit our application whose dataset consists of timestamped sensor event triggers as depicted in Fig. 1, e.g., On 2010-11-4 at 19:48:25.951116, the sensor M015 got switched ON. The first step is to reformat the data in a more usable form. First, the dataset is converted to a set of binary time series, a series for each sensor. At each time stamp of the dataset, the signal value $(0 / 1)$ of each sensor is calculated, based on its previous value and the current event. Thus, the dataset is converted from an event-based to a signal-based dataset. Then, all-zeros rows were deleted as we are interested in the relation between positively triggered sensors. The resulted transformation can be seen on Fig. 2(a).

In a market basket analysis, transactional datasets are analysed to discover which items are likely to be bought together. Similarly, in AAL, we would like to know which sensors are likely to be ON simultaneously. Even in single-resident homes, simultaneously ON sensors in different locations can 


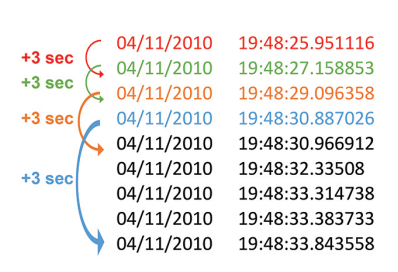

$\ldots$

\begin{tabular}{|cccccc|} 
M015 & M018 & M019 & M021 & M022 & M023 \\
\hline 1 & 0 & 0 & 0 & 0 & 0 \\
\hline 1 & 0 & 1 & 0 & 0 & 0 \\
\hline \hline 1 & 1 & 1 & 0 & 0 & 0 \\
\hline \hline 1 & 1 & 1 & 1 & 0 & 0 \\
1 & 1 & 0 & 1 & 0 & 0 \\
\hline 1 & 1 & 0 & 1 & 1 & 0 \\
0 & 1 & 0 & 1 & 1 & 0 \\
0 & 0 & 0 & 1 & 1 & 0 \\
0 & 0 & 0 & 1 & 1 & 1 \\
\hline
\end{tabular}

(a)

$\begin{array}{llcccccc}\text { 04/11/2010 } & 19: 48: 25.951116 & \text { M015 } & \text { M018 } & \text { M019 } & \text { M021 } & \text { M022 } & \text { M023 } \\ \text { 04/11/2010 } & 19: 48: 27.158853 & 1 & 0 & 1 & 0 & 0 & 0 \\ 04 / 11 / 2010 & 19: 48: 29.096358 & 1 & 1 & 1 & 0 & 0 & 0 \\ 04 / 11 / 2010 & 19: 48: 30.887026 & 1 & 1 & 1 & 1 & 0 & 0 \\ & & & & & & & 1\end{array}$

(b)

Fig. 2. Data aggregation using sliding window of size 3 seconds

be observed due to the switch-off delay time of motion sensors or due to the overlapping detection areas of those sensors. We are also interested to know which sensors are usually triggered within a few seconds from each other. To take into account the temporal correlations, we consider that if a sensor is ON within $t$ seconds from another sensor then they are considered as happening simultaneously. Thus, a time-based sliding window is then used to aggregate the sensor data into a set of transactions as in Fig. 2(b).

\section{B. Association Rules Mining}

In an attempt to find the strong association rules that reflects the correlations between sensors, the use of rule mining with different measures were investigated. The first technique uses the typical measures for association rule mining which are the support and confidence of a rule. The minimum support and minimum confidence values should be determined by the designer to control the quality of the obtained association rules. The support calculates the probability of finding two or more simultaneous positively triggered sensors.

As the use of different areas in the apartment may not be equally distributed, some sensors may be triggered much less often than others, consequently their support will be relatively low in comparison to others, and thus, they will not appear in the extracted rules as they did not exceed the minimum support value. Consequently, a new measure termed relative support is calculated as defined in equation 4 . The second technique investigate the use of the relative support and confidence as evaluation metrics for the association rules mining.

Rel. $\operatorname{Support}(X \rightarrow Y)=$

$\mid$ Transactions containing $\mathrm{X} \& \mathrm{Y} \mid$

$\overline{\operatorname{Min}(\mid \text { Transactions for each item in } \mathrm{X} \text { or } \mathrm{Y} \mid)}$

For example, the relative support of the rule M4,M5 $\rightarrow$ M7, is calculated as follows:

$$
\begin{aligned}
\text { Rel. Support }(M 4, M 5 \rightarrow M 7)= & \frac{\text { Trans }_{4,5 \& 7}}{\operatorname{Min}\left(\text { Trans }_{4}, \text { Trans }_{5}, \text { Trans }_{7}\right)}
\end{aligned}
$$

where Trans $4,5 \& 7$ is the number of transactions in which the sensors M4, M5 and M7 appear together, and Trans 4 , Trans 5 and Trans $_{7}$ are the number of transactions containing M4, M5 and M7, respectively.

\section{CASE STUDY}

\section{A. Dataset}

The proposed approach has been evaluated on the publicly available Aruba CASAS dataset [12], which was collected from a single-resident apartment for 6 months. The apartment is equipped with 31 motion sensors, 4 contact door sensors and 5 temperature sensors. However, only the motion and contact sensors were included in our experiments. Also, there is one door contact sensor that never triggered any event in the recorded dataset. Thus, in total we have used the data from 31 motion sensors and 3 contact sensors which results in a dataset of 1316981 sensor triggers. The training data used for finding the correlations is $50 \%$ of the dataset. The other $50 \%$ of the data is left to be used for the validation of a sensor failure detection system based on the extracted correlations; however, this is not addressed in this paper. Also, it is assumed that the recorded dataset does not have faulty sensors triggers.

\section{B. Experiments}

In order to evaluate the techniques proposed in the previous section, two experiments were conducted using MATLAB 2017b software. Standard support experiment ( Experiment A) uses the first technique which implements association rule mining with minimum support values of $0.5 \%, 1 \%$ and $1.5 \%$, minimum confidence values of $60 \%$,

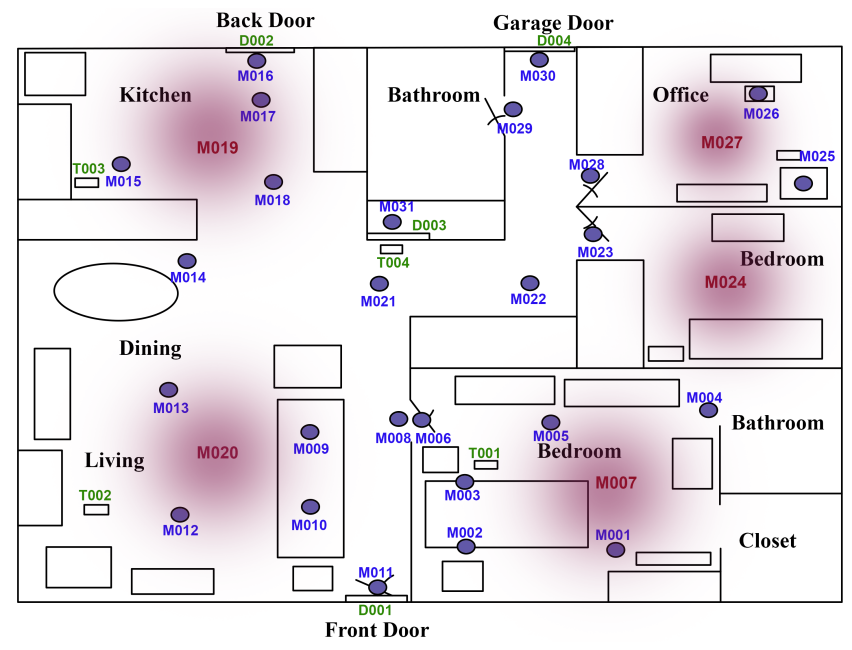

Fig. 3. Aruba CASAS floor plan [12] 
$80 \%, 90 \%$ and $100 \%$, and sliding window sizes of 3,5 , $8,10,15,30$ and 45 seconds. Relative support experiment (Experiment B) uses the second technique which depends on the minimum relative support instead of the minimum support and experimented with the values of $15 \%, 20 \%, 25 \%$, $35 \%$ and $45 \%$, and the same minimum confidence and sliding window parameters.

The objective of these experiments is to extract meaningful association rules that have as many as possible of the employed sensors appearing in at least one of the consequent parts of the extracted rules so that most sensors could be checked for faulty behaviour. An example of the outputted association rules is as follows: M6, M8 $\rightarrow$ M20 (Support: $4.5942 \%$, Confidence: $92.9159 \%$ ). This rule means that, according to the given dataset, $92.9159 \%$ of the times the sensors M6 and M8 were active, the sensor M20 was also active. From a fault detection perspective, it would also means that, if during a real-time monitoring, the sensors M6 and M8 are active and the sensor M20 is not activated within the size of sliding window used in the data preprocessing, then it is highly probable that any of these sensors is faulty.

In the next subsection, several combinations of the abovementioned parameters will be evaluated and compared with respect to the number of extracted rules, the number of sensors present in the consequent part and the ratio of sensors present in the consequent part to the number of extracted rules.

\section{Results}

The number of association rules obtained from each experiment, the number of the sensors present in the consequent part of those rules and the ratio of sensors present in the consequent part to the number of extracted rules were plotted on Fig. 4, Fig. 5 and Fig. 6, respectively.

First, as shown in Fig. 4, in all experiments the number of obtained rules increases roughly linearly as the sliding window size is increased from 3 to 10 seconds included, then afterwards the number increases exponentially even more drastically. On the other hand, as shown in Fig. 5, after 10 seconds the number of sensors in the consequent part of rules does not increase in the same rate of increase of number of rules. However, as shown in Fig. 6, for a sliding window larger than 10 seconds, a dramatic drop occurs in the ratio of the number of sensors to the number of rules. As a first result, it can be concluded that for a sliding window larger than 10 seconds, the drastic increase in the number of association rules is not useful as it does not permit to extract more new rules that covers the sensors that have been missing in the consequent parts.

In the standard support experiment, the graphs plotted in Fig. 4 clearly show that the trend is almost consistent within the sub-experiments; at each specific minimum support, the number of rules and consequent sensors increase for each value of minimum confidence as the sliding window size increase. The trend is also consistent on the global view of the sub-experiments; as the minimum support decreases, the number of association rules increase (see Fig. 4) as well as the number of sensors in consequent part (see Fig. 5). However, the latter one is increasing less rapidly, as depicted in Fig. 6, where the ratio of sensors to rules decreases as the minimum support decreases.

Similarly to the standard support experiment, in the relative support experiment as the minimum support decreases the number of association rules and consequent sensors

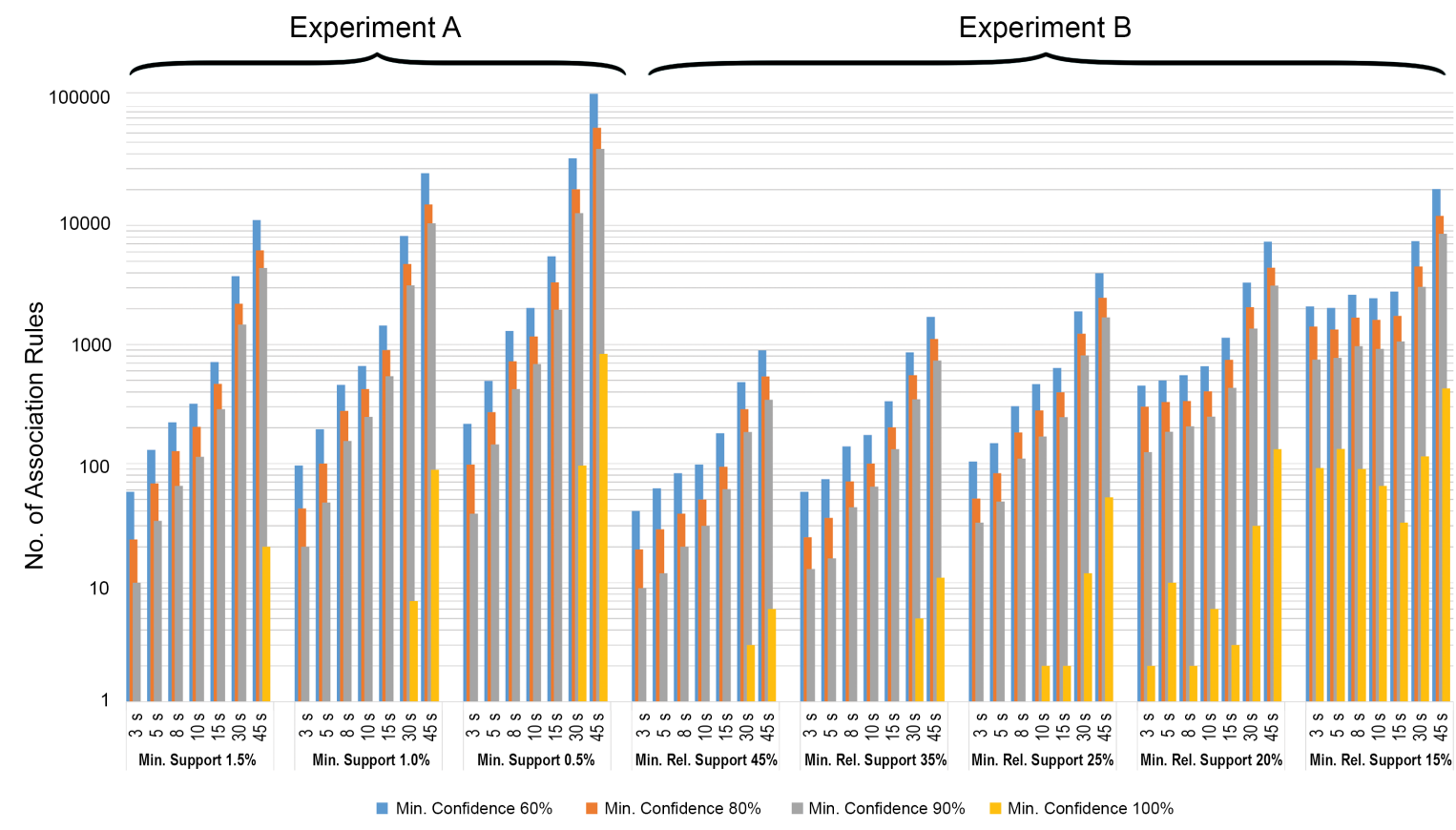

Fig. 4. Number of association rules w.r.t. minimum support/relative support, minimum confidence and sliding window size 


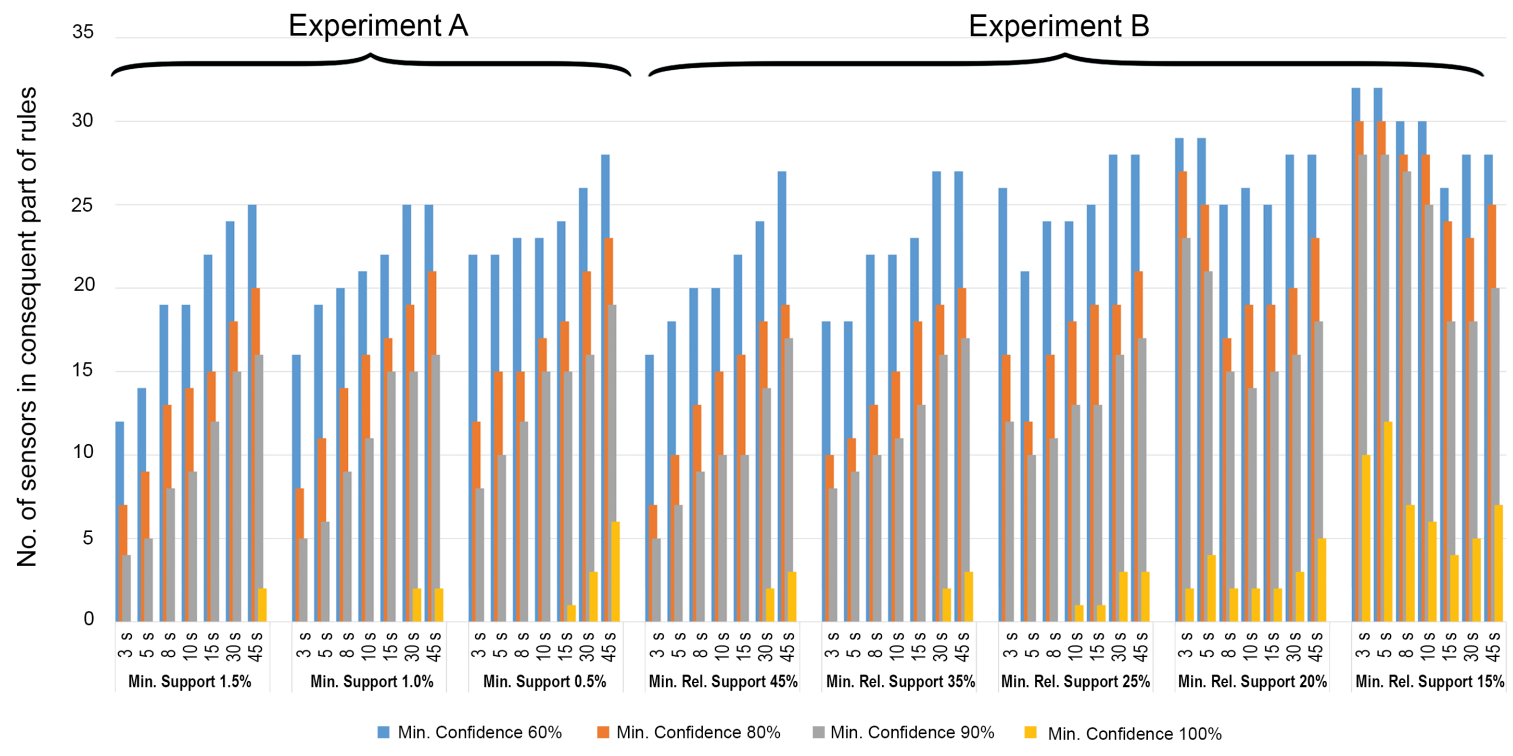

Fig. 5. Number of sensors in the consequent part of association rules

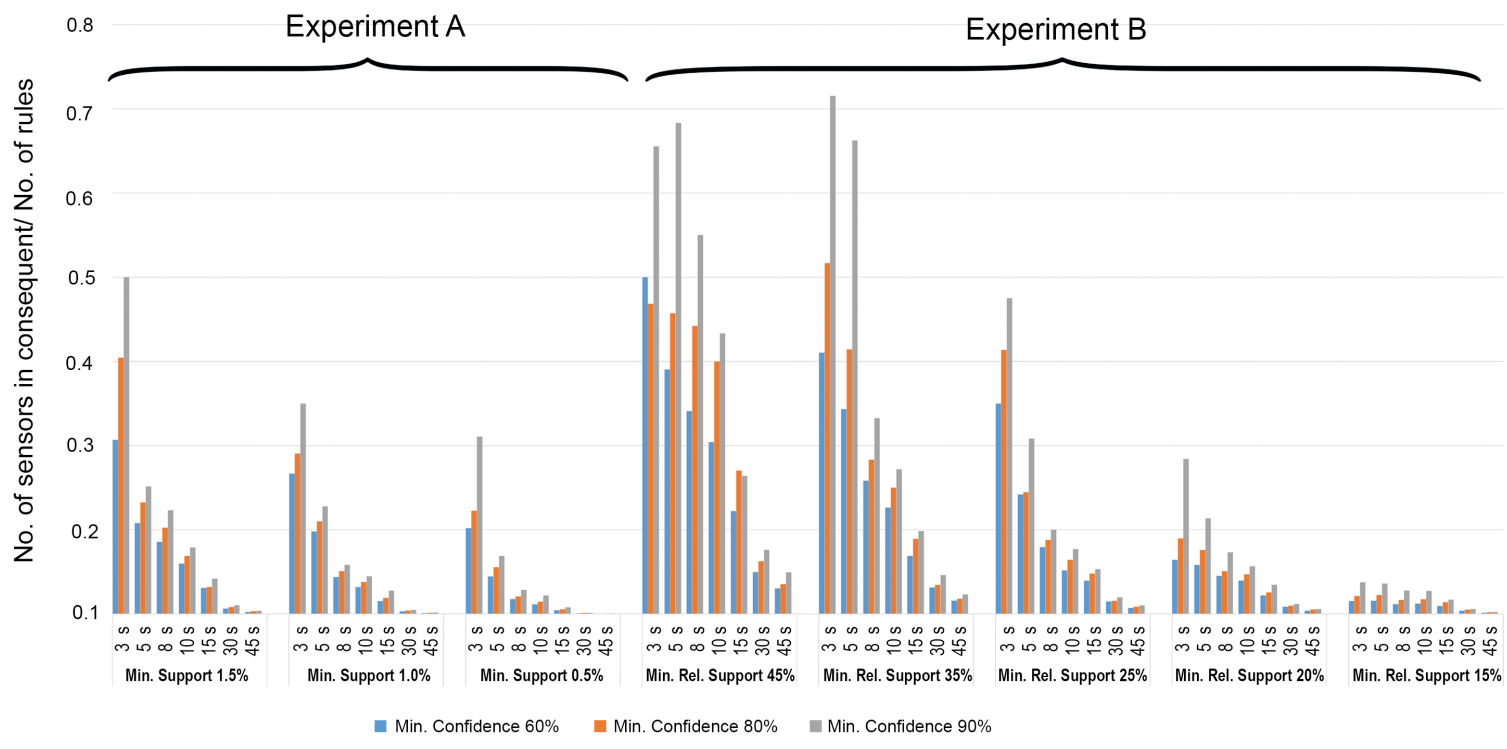

Fig. 6. Ratio of the number of sensors in the consequent part of association rules to the number of rules

increase. However, the plots of the relative support experiment show that the trend within the sub-experiments is inconsistent, for example, at minimum relative support of $20 \%$ the number of consequent sensors at a sliding window size of 10 seconds that have a confidence of $100 \%$ is greater than that at 8 seconds, while at minimum relative support of $15 \%$ the same parameter is less at 10 seconds than that at 8 seconds (see Fig. 5). This could be due to the fact that each of the numerator and denominator of the relative support equation increases in different rates according to the switchoff delays of motion sensors and the resident's behaviour, changing the relative support of each itemset.
As observed in Fig. 6, the ratio of consequent sensors to the number of rules that have a confidence of $90 \%$ or more is always the highest in all sub-experiments compared to the other minimum confidence values. By setting the minimum relative support to $35 \%$, sliding window size to 3 seconds and minimum confidence to $90 \%$, the resulted ratio of consequent sensors to rules is 0.615 . However, the number of consequent sensors is only 8 . Therefore, such rules are not sufficient to discover faulty sensors in an apartment that deploys 34 sensors. As illustrated, judging the best parameters for achieving good results in association rules from investigating either one or two of Fig. 4, 5 and 6 is not 


$\begin{array}{ll}\text { Rule } & (\text { Support, Confidence, Lift) } \\ \text { M4,M5 -> M7 } & (1.8598 \%, 99.7798 \%, 7.0076) \\ \text { M27,M28 -> M26 } & (0.44116 \%, 97.0335 \%, 24.9976) \\ \text { M7,M8 -> M6 } & (2.2994 \%, 77.7066 \%, 13.104) \\ \text { M28,M31 -> M29 } & (0.13819 \%, 96.5847 \%, 63.3015) \\ \text { M10,M13 - M9 } & (1.8377 \%, 76.0495 \%, 3.7885) \\ \text { M15,M16 -> M19 } & (1.318 \%, 96.5078 \%, 3.8882) \\ \text { M5 -> M7 } & (5.7343 \%, 94.9325 \%, 6.6672) \\ \text { M6,M8 -> M20 } & (4.5942 \%, 92.9159 \%, 3.0962) \\ \text { M10,M12,M13 -> M20 } & (1.2897 \%, 92.0608 \%, 3.0677) \\ \text { M4,M7 -> M5 } & (1.8598 \%, 64.6531 \%, 10.7034) \\ \text { D4,M29 -> M30 } & (0.59519 \%, 91.4964 \%, 50.4254) \\ \text { M21,M23 -> M22 } & (1.4179 \%, 90.5279 \%, 13.6379) \\ \text { D2,M19 -> M16 } & (0.11063 \%, 90.4153 \%, 20.0021) \\ \text { D1,M11 -> M20 } & (0.15969 \%, 78.7091 \%, 2.6228) \\ \text { M29,M30 -> D4 } & (0.59519 \%, 68.4423 \%, 45.958)\end{array}$

Fig. 7. Sample of the obtained rules at $25 \%$ min. relative support, $60 \%$ min. confidence and sliding window size of 3 seconds

sufficient, the three figures should be considered together to determine the best trade-off among the parameters, as will be discussed in the next section.

\section{Discussion}

Overall, the relative support experiment permits to extract more consequent sensors within less number of functionally redundant rules than the standard support experiment. This shows that using the relevant support of rule is more beneficial for our application. This can be explained by the fact that the AAL datasets are usually unbalanced: some sensors are triggered much more often than others; yet, infrequent triggered sensors may be highly correlated.

Pursuing a trade-off between the number of meaningful association rules and consequent sensors, a minimum relative support of $25 \%$, combined with a sliding window size of 3 seconds and a minimum confidence of $60 \%$ appear to be the best values for extracting strong correlations between sensors that could be used to detect sensor failures. Those parameters produce 104 rules that contains 26 different sensors in the consequent part of the association rules. 32 out of the 104 rules have a confidence of $90 \%$ or more; 12 sensors out of the 26 consequent sensors are included in those $90 \%$ confidence rules. In general, this means that for some sensors it would be easy to extract relations with a few rules, but others may require many more rules or are even impossible to correlate for a given configuration of these sensors positioning in an apartment. A sample of the obtained rules is shown in Fig. 7 , the lift value for all the rules is always greater than 1 .

By checking the extracted association rules and the apartment layout, logically correct correlations could be obtained between the different sensors. As a consequence, a sensor failure detection for Ambient Assisted Living could rely on those rules to flag a fail-stop or non-fail-stop sensor failure. Those rules could be exploited to build a probabilistic model for sensors' triggers which can be used for fault detection. As the number of rules with $100 \%$ confidence is relatively small, it is not possible, after a positive/negative trigger observation of a sensor, to guarantee with certitude whether the sensor is faulty or not. Yet, after a sequence of highly probable unexpected events (confidence $>80 \%$ ), the confidence in the diagnostic can be increased.

\section{CONCLUSION AND FUTURE WORK}

This paper proposed the use of association rule mining to find the correlations between binary event-triggered sensors deployed in Ambient Assisted living environment. Two techniques were implemented, one using support and the other using relative support, and compared on a case study. The criteria for obtaining the association rules of interest were discussed. The proposed approach, using the relative support of a rule, permits to obtain interesting correlations between sensors from an unlabelled dataset, with no need for prior expert knowledge.

As future work, those correlations could be used for the detection of fail-stop and non-fail-stop sensor failures and/or fault tolerance in Ambient Assisted Living. A limitation for the proposed system is that some of the deployed sensors do not have rules that enable us to check on their faulty behaviour. This point will be considered in the future work, especially in relation to existing works on automatic placement of sensors for ADL recognition [13]. Also, a sensor failure detection system will be developed based on the obtained rules and real-time experiments will be conducted with injecting various types of failures to evaluate the performance of detecting failures.

\section{REFERENCES}

[1] World Health Organization, "Global strategy and action plan on ageing and health," 2016.

[2] M. Flöck, Activity monitoring and automatic alarm generation in AALenabled homes. Logos Verlag Berlin GmbH, 2010.

[3] K. Kapitanova, E. Hoque, J. A. Stankovic, K. Whitehouse, and S. H. Son, "Being smart about failures: assessing repairs in smart homes," in Proc. of the 2012 ACM Conf. on Ubiquitous Computing, 2012, pp. 51-60.

[4] S. Munir and J. A. Stankovic, "Failuresense: Detecting sensor failure using electrical appliances in the home," in Mobile Ad Hoc and Sensor Systems (MASS), 2014 IEEE 11th Int. Conf. on, 2014, pp. 73-81.

[5] J. Ye, G. Stevenson, and S. Dobson, "Detecting abnormal events on binary sensors in smart home environments," Pervasive and Mobile Computing, vol. 33, pp. 32-49, 2016.

[6] M.-H. Amri, D. Aubry, Y. Becis, and N. Ramdani, "Robust fault detection and isolation applied to indoor localization," Special issue: 9th IFAC Symposium on Fault Detection, Supervision andSafety for Technical Processes SAFEPROCESS 2015, vol. 48, no. 21, pp. 440445, 2015.

[7] P. A. Kodeswaran, R. Kokku, S. Sen, and M. Srivatsa, "Idea: A system for efficient failure management in smart iot environments," in Proc. of the 14th Int. Conf. on Mobile Systems, Applications, and Services. ACM, 2016, pp. 43-56.

[8] J. Ye, G. Stevenson, and S. Dobson, "Using temporal correlation and time series to detect missing activity-driven sensor events," in Pervasive Computing and Communication Workshops (PerCom Workshops), 2015 IEEE Int. Conf. on, 2015, pp. 44-49.

[9] R. Agrawal, T. Imieliński, and A. Swami, "Mining association rules between sets of items in large databases," in Acm sigmod record, vol. 22, no. 2. ACM, 1993, pp. 207-216.

[10] A. Rajak and M. K. Gupta, "Association rule mining: applications in various areas," in Proc. of Int. Conf. on Data Management, 2008, pp. 3-7.

[11] T. Karthikeyan and N. Ravikumar, "A survey on association rule mining," Int. Journal of Advanced Research in Computer and Communication Engineering, vol. 3, no. 1, pp. 2278-1021, 2014.

[12] "Casas datasets," http://ailab.wsu.edu/casas/datasets/, accessed: 9-32018.

[13] M. P. Fanti, G. Faraut, J.-J. Lesage, and M. Roccotelli, "An integrated framework for binary sensor placement and inhabitants location tracking," IEEE Trans. on Systems, Man, and Cybernetics: Systems, 2016. 